\title{
GAUSSIAN-MARKOV PROCESSES AND \\ A BOUNDARY VALUE PROBLEM $\left({ }^{1}\right)$
}

\author{
BY \\ J. A. BEEKMAN
}

I. Summary. One purpose of this article is to obtain an integral representation for a solution of the following boundary value problem:

$$
\begin{aligned}
A(t) \frac{\partial^{2} r}{\partial y^{2}}-B(t) \frac{\partial}{\partial y}[y r]-\frac{\partial r}{\partial t}+\theta(y, t) r & =0, \\
0 & \leqq s<t<T, \quad y \in\{(-\infty, \infty)-E\}
\end{aligned}
$$

where $E$ is a finite set of points, $A(t)>0, A^{\prime}(t)$, and $B^{\prime}(t)$ are continuous on $0 \leqq t \leqq T$, and $\theta(y, t)$ and $\theta_{y}(y, t)$ are bounded, continuous complex valued functions for $0 \leqq t \leqq T, y \in\{(-\infty, \infty)-E\}$.

(1.2) $\lim _{t \rightarrow s^{+}} \int_{-\infty}^{\infty} g(x) r(x, s \mid y, t) d x=g(y)$ for every bounded continuous $g$.

$$
\lim _{|y| \rightarrow \infty} r(x, s \mid y, t)=0 .
$$

(1.4) $\partial r / \partial y, \partial^{2} r / \partial y^{2}, \partial r / \partial t$ continuous for $0 \leqq s<t<T, \quad y \in\{(-\infty, \infty)-E\}$.

$\partial r / \partial y$ is also continuous if $y \in E$.

We shall also show our solution satisfies a backward version of (1.1)-(1.4), and is unique. The solution will involve an expectation over the sample functions of a Gaussian-Markov process.

An $n$-dimensional version of a similar system has been studied by M. Rosenblatt [21] and D. Ray [20], for the Wiener process where $B(t)=0$. M. Kac studied the Wiener version in [15], as did R. H. Cameron in [5], subject to different boundary conditions. A backward time version of (1.1) with different boundary conditions was studied by the author in [3], as an underlying theorem for generalized Schroedinger equations. The need to develop forward time forms of such equations, and to weaken the hypotheses on the potential $\theta(y, t)$ helped motivate this paper. R. K. Getoor considered (1.1) for stationary Markov processes in [11], [12]. The principal reference is D. A. Darling and A. J. F. Siegert [8]. They proved that if:

$$
r(x, s \mid y, t)=E\left\{\exp \left[i \xi \int_{s}^{t} \phi[x(\tau), \tau] d \tau\right] \mid x(s)=x, x(t)=y\right\} p(x, s ; y, t)
$$

Presented to the Society, November 26, 1965; received by the editors March 17, 1966.

(1) This research was partially supported by the National Science Foundation through grant NSF GP-4570. 
where the expectation is over the paths of a Markov process, and $p(x, s ; y, t)$ is the transition density function of the process, then $r$ is the unique solution of a pair of integral equations. This was done for a general Markov process. They also proved that if a principal solution existed for (1.1), then it had to satisfy the pair of integral equations and hence was the Markovian function. This paper is restricted to Gaussian-Markov processes and shows that for such a process the corresponding $r(x, s \mid y, t)$ does satisfy (1.1)-(1.4) as well as the adjoint differential equation and corresponding conditions. Hence we can conclude that the solution is unique.

The second purpose of the paper is the application of the theorems to calculating the distributions of functionals defined on Gaussian-Markov processes. The coefficients $A(t)$ and $B(t)$ for three such processes are quoted and the distribution for a random walk with two absorbing barriers for the Ornstein-Uhlenbeck process is considered.

II. Introduction. Let $x(t), a \leqq t \leqq b$ be a Gaussian-Markov stochastic process with transition density function

$$
\begin{aligned}
p(x, s ; y, t) & =\frac{\partial}{\partial y} P[x(t) \leqq y \mid x(s)=x] \\
& =\{2 \pi A(s, t)\}^{-1 / 2} \exp \left\{-\frac{[y-(v(t) / v(s)) x]^{2}}{2 A(s, t)}\right\}
\end{aligned}
$$

where

$$
\begin{gathered}
A(s, t) \equiv\left[u(t) v(t)-\frac{u(s)}{v(s)} v^{2}(t)\right], \quad a \leqq s \leqq t \leqq b, \\
u(t) \geqq 0, \quad v(t)>0, \quad a \leqq t \leqq b, \\
u^{\prime \prime}(t), v^{\prime \prime}(t) \text { exist, and are continuous on }[a, b] \\
{\left[v(t) u^{\prime}(t)-u(t) v^{\prime}(t)\right]>0, \quad a \leqq t \leqq b .}
\end{gathered}
$$

Since

$$
\begin{aligned}
\lim _{t \rightarrow s+} P[x(t) \leqq y \mid x(s)=x] & =1, & & y>x \\
& =0, & & y<x
\end{aligned}
$$

with probability one, the transition density function determines a separable and measurable stochastic process $x(\tau), s \leqq \tau \leqq t$ with $x(s)=x, x(t)=y$ with probability one. The $u$ and $v$ functions specify the process which is not subject to the restriction $x(t)=y$. This follows since they determine the mean and covariance functions of that process. Condition (2.4) insures the continuity of almost all sample functions [9]. It is well known that $p(x, s ; y, t)$ satisfies the two diffusion equations of Fokker-Planck, which are (1.1) and (3.3) with $\theta \equiv 0, p$ replacing $r$, and coefficients (3.1) and (3.2). 


\section{Statement of theorems.}

THEOREM 1. Assume that $\{x(t), 0 \leqq t<\infty\}$ is a Gaussian-Markov stochastic process with transition function (2.1) subject to (2.2)-(2.5). Assume that $\theta(y, \tau)$ and $\theta_{y}(y, \tau)$ are bounded and continuous complex valued functions in $S$ :

$$
0 \leqq \tau \leqq T<\infty, \quad y \in\{(-\infty, \infty)-E\}
$$

where $E$ is a finite set of points.

Then the function $r(x, s \mid y, t)$ given by (1.5) (with $\theta$ replacing $i \xi \phi)$ uniquely satisfies (1.1)-(1.4), as a function of $y$ and $t$, with

$$
\begin{aligned}
& A(t)=\left[v(t) u^{\prime}(t)-u(t) v^{\prime}(t)\right] / 2, \\
& B(t)=v^{\prime}(t) / v(t), \quad 0 \leqq t \leqq T .
\end{aligned}
$$

Furthermore $r(x, s \mid y, t)$ uniquely satisfies (as a function of $x$ and $s$ ):

$$
A(s) \frac{\partial^{2} r}{\partial x^{2}}+x B(s) \frac{\partial r}{\partial x}+\frac{\partial r}{\partial s}+\theta(x, s) r=0
$$

for $0<s<t \leqq T, x \in\{(-\infty, \infty)-E\}$.

$$
\lim _{s \rightarrow t-} \int_{-\infty}^{\infty} g(y) r(x, s \mid y, t) d y=g(x) \text { for every continuous bounded } g .
$$

$$
\lim _{|x| \rightarrow \infty} r(x, s \mid y, t)=0
$$

$$
\frac{\partial r}{\partial x}, \frac{\partial^{2} r}{\partial x^{2}}, \frac{\partial r}{\partial s} \text { are continuous for } 0<s<t \leqq T, \quad x \in\{(-\infty, \infty)-E\}
$$

$\partial r / \partial x$ is also continuous if $x \in E$.

RemarK. The coefficients (3.1) and (3.2) appear quite specific. However, if an equation of the form (1.1) is given with $A(t)>0, A^{\prime}(t)$ and $B^{\prime}(t)$ continuous on $0 \leqq t \leqq T$, one can use the $A(t)$ and $B(t)$ to construct $u(t)$ and $v(t)$ functions which satisfy (2.3), (2.4), (2.5), (3.1), and (3.2). (See [3, p. 805].) Then if $\theta(y, t)$ is as in Theorem 1, the $r$ function with the constructed $u$ and $v$ functions satisfies (1.1)(1.4), (3.3)-(3.6).

THEOREM 2. Assume that $\{x(t), 0 \leqq t<\infty\}$ satisfies the hypotheses of Theorem 1 , and has a stationary transition density function. Assume that $\theta(y, \tau)=-u V(y)$, $-\infty<y<\infty, u>0$, where $V$ is a nonnegative function for which $V(y)$ and $V^{\prime}(y)$ are continuous and bounded, $y \notin E, E$ a finite set of points. Let $Q(y, t)=r(0,0 \mid y, t)$.

If $\hat{Q}(y)=\int_{0}^{\infty} e^{-\lambda t} Q(y, t) d t, \lambda>0, \hat{Q}$ is the unique solution of

$$
A \frac{d^{2} \hat{Q}}{d y^{2}}-B \frac{d}{d y}(y \hat{Q})-u V(y) \hat{Q}=\lambda \hat{Q}, \quad y \neq 0, y \notin E
$$


where $A(>0)$ and $B$ are the constants in the forward diffusion equation.

$$
\begin{gathered}
\hat{Q}^{\prime}(0+)-\hat{Q}^{\prime}(0-)=-1 / A, \\
\lim _{|y| \rightarrow \infty} \sqrt{|y|} \hat{Q}(y)=0, \\
\hat{Q}(y) \text { continuous for all } y . \\
\hat{Q}^{\prime}(y) \text { continuous for } y \neq 0 . \\
\hat{Q}^{\prime \prime}(y) \text { continuous for } y \neq 0, y \notin E . \\
\left|\hat{Q}^{\prime}(y)\right|<C+D / \lambda, C \text { and } D \text { constants, } y \neq 0 .
\end{gathered}
$$

REMARK. The proofs of the theorems are given in $\S \mathrm{VI}$.

IV. Uses of the theorems. The theorems of this paper can be used in calculating the distribution functions of functionals $\int_{s}^{t} \phi[x(\tau), \tau] d \tau$ defined on the trajectories of a Gaussian-Markov process.

If we let

$$
\sigma(\alpha ; s, x)=P\left[\int_{s}^{t} \phi[x(\tau), \tau] d \tau<\alpha \mid x(s)=x\right]
$$

then

$$
\begin{aligned}
\int_{-\infty}^{\infty} \exp (i \xi \alpha) d_{\alpha} \sigma(\alpha ; s, x) & =E\left\{\exp \left[i \xi \int_{s}^{t} \phi[x(\tau), \tau] d \tau\right] \mid x(s)=x\right\} \\
& =\int_{-\infty}^{\infty} r(x, s \mid y, t) d y
\end{aligned}
$$

Thus the integration of the $r$ function of Theorem 1 yields the characteristic function of the desired distribution function which can be inverted to yield $\sigma$.

In some cases the partial differential equation satisfied by $r$ can be converted to a corresponding Sturm-Liouville equation which can be explicitly solved for $r$. See E. Wong [25]. This reference also relates to the work of Darling and Siegert [8]; it does not consider the existence problems discussed in this paper.

The $Q$ function can be used to give an expression for the distribution function for a random walk with two absorbing barriers for any Gaussian-Markov process.

$$
\begin{aligned}
P & \equiv P\left[-b<\inf _{0 \leqq s \leqq t} x(s) \leqq \sup _{0 \leqq s \leqq t} x(s)<a \mid x(0)=0\right] \\
& =\lim _{u \rightarrow \infty} \int_{-\infty}^{\infty} Q(y, t) d y
\end{aligned}
$$

where $Q(y, t)$ satisfies (1.1) subject to conditions (6.17), (1.3), (1.4) with

$$
\begin{array}{rlrl}
\theta(y, t)=\theta_{u}(y) & =0, & & -b<y<a, a>0, b>0 \\
& =-u, & y<-b, \quad y>a
\end{array}
$$

and $E=\{-b, a\}$. 
This follows since the continuity of the sample functions gives

$$
P=\lim _{u \rightarrow \infty} E\left\{\exp \left[\int_{0}^{t} \theta_{u}[x(s)] d s\right] \mid x(0)=0\right\}
$$

This idea was used by M. Kac in [15] and [16]. The expression for $P$ includes as subcases

$$
P\left[\sup _{0 \leqq s \leqq t} x(s)<a \mid x(0)=0\right]
$$

and

$$
P\left[\max _{0 \leqq s \leqq t}|x(s)|<a \mid x(0)=0\right] .
$$

The $r$ function will be used in developing generalized Feynman integrals similar to those of [3]. It is expected that those integrals will satisfy a forward time version of the generalized Schroedinger equation (equation (6.2) of [3]). Fewer restrictions on the potential $\theta(x, t)$ will be used than in [3].

V. An example. Only one example will be considered in detail. However, since the coefficients of equation (1.1) are basic to all examples, the coefficients $A(t)$ and $B(t)$ are given for three Gaussian-Markov processes.

EXAMPLE 1. Wiener process: $u(t)=t, v(t)=1, A(t)=1 / 2, B(t)=0$.

EXAMPLE 2. $u(t)=t, v(t)=1-t\left(1+4 c^{2}\right), 0 \leqq t<1 /\left(1+4 c^{2}\right), c \geqq 0, A(t)=1 / 2$, $B(t)=-\left(1+4 c^{2}\right) /\left[1-t\left(1+4 c^{2}\right)\right]$. This process was first studied by J. L. Doob [9], and M. Kac [14], with $c=0$. In reference [23] I. Vincze considered a process with this covariance and mean function $E\{X(t)\}=-2 c t$. Expectations on this process are related to the zero mean function process by the equation

$$
E\{F[x]\}=E\{F[Y(\cdot)-2 c(\cdot)]\} .
$$

EXAMPLE 3. Ornstein-Uhlenbeck process [19]: $u(t)=\sigma^{2} e^{\beta t}, v(t)=e^{-\beta t}, \sigma^{2}>0$, $\beta>0, A(t)=2 \sigma^{2} \beta, B(t)=-\beta$.

We now consider the distribution $P$ defined by (4.3) for the Ornstein-Uhlenbeck process. Part of the analysis is similar to that employed in [8] for the double transform of the distribution of the functional $1 / 2 \int_{0}^{t} \operatorname{sgn} x(s) d s$ for this process. By Theorem 2 and an extension of (4.3)-(4.4) we must solve

$$
\sigma^{2} \beta \frac{d^{2} \hat{Q}}{d y^{2}}+\beta \frac{d(y \hat{Q})}{d y}+[-u V(y)-\lambda] \hat{Q}=0, \quad y \notin\{-b, 0, a\}
$$

with

$$
\begin{array}{rlrl}
V(y) & =0, & -b & <y<a, a>0, b>0 \\
& =1, \quad y<-b, \quad y>a
\end{array}
$$

where $\hat{Q}$ is subject to (3.8)-(3.11).

For simplicity we will let $\sigma^{2}=1$. The retention of $\beta$ is because it may be interpreted to be the reciprocal of the friction coefficient, and for sufficiently large 
values of the friction coefficient and $t$, the Ornstein-Uhlenbeck process serves as a model for the Brownian motion of an elastically bound particle [13]. (Also see [19].)

Dividing by $\beta$, and using a transformation of variables, solutions to (5.1) are found to be $\hat{Q}=D_{k}( \pm y) \exp \left(-y^{2} / 4\right)$ where $k=(-u V(y)-\lambda) / \beta$ and $D_{v}(z)$ is the Weber function [24].

Let $\lambda^{\prime}=\lambda / \beta$ and $u^{\prime}=u / \beta$. To obtain Green's solution we consider $\hat{Q}$ of the form

$$
\begin{array}{rlrl}
\hat{Q} & =c D_{-u^{\prime}-\lambda^{\prime}}(y) \exp \left(-y^{2} / 4\right), \quad y>a, & \\
& =d D_{-\lambda^{\prime}}(y) \exp \left(-y^{2} / 4\right)+e D_{-\lambda^{\prime}}(-y) \exp \left(-y^{2} / 4\right), & & 0<y<a, \\
& =f D_{-\lambda^{\prime}}(y) \exp \left(-y^{2} / 4\right)+g D_{-\lambda^{\prime}}(-y) \exp \left(-y^{2} / 4\right), & & -b<y<0, \\
& =h D_{-u^{\prime}-\lambda^{\prime}}(-y) \exp \left(-y^{2} / 4\right), \quad y<-b . &
\end{array}
$$

Using the asymptotic expansion for $D_{k}( \pm y)$, one sees that

$$
\lim _{|y| \rightarrow \infty} \sqrt{|y|} D_{k}( \pm y) \exp \left(-y^{2} / 4\right)=0
$$

which establishes (3.9). Next we use the formula for $(d / d y)\left[\exp \left(-y^{2} / 4\right) D_{k}(y)\right]$ and an integral representation for $D_{k}(y)$ to verify (3.11). Conditions (3.8) and (3.10) will be obtained by suitable choices of the constants in (5.2).

The conditions imposed on $\hat{Q}$ and $\hat{Q}^{\prime}$ at $-b, 0$, and $a$, yield six equations in the six unknowns. The results are complicated and only intermediate to their results as the parameter $u \rightarrow \infty$, so will not be recorded.

By Theorem 2, we have now uniquely specified $\hat{Q}(y)$.

By Fubini's Theorem, we have

$$
\int_{-\infty}^{\infty} \hat{Q}(y) d y=\int_{0}^{\infty} e^{-\lambda t} E\left\{\exp \left[\int_{0}^{t} \theta_{u}[x(s)] d s\right] \mid x(0)=0\right\} d t
$$

By dominated convergence and (4.4),

$$
\lim _{u \rightarrow \infty} \int_{-\infty}^{\infty} \hat{Q}(y) d y=\int_{0}^{\infty} e^{-\lambda t} P d t
$$

Using an asymptotic expression for $D_{\sqrt{ }}(z)$ as $|\sqrt{ }| \rightarrow \infty$, [6], one can show that $(\delta>0)$

$$
\lim _{u \rightarrow \infty} \int_{\delta}^{\infty} \exp \left(-y^{2} / 4\right) D_{-u^{\prime}-\lambda^{\prime}}(y) / D_{-u^{\prime}-\lambda^{\prime}+1}(\delta) d y=0 .
$$

Using equations (10) and (4) of [2, p. 117], and the duplication formula of Legendre for gamma functions, [18, p. 485], one can show that

$$
D_{\sqrt{ }}(z) D_{\sqrt{ }+1}(-z)+D_{\sqrt{ }}(-z) D_{\sqrt{ }+1}(z)=2 D_{\sqrt{ }}(0) D_{\sqrt{ }+1}(0)
$$

for complex $z$ and $\sqrt{ }<0$. 
Using (5.4), (5.5), and (5.6), one obtains the new result

$$
\begin{aligned}
& \int_{0}^{\infty} e^{-\lambda^{\prime} w} P\left[-b<\inf _{0 \leqq s \leqq w / \beta} x(s) \leqq \sup _{0 \leqq s \leqq w / \beta} x(s)<a \mid x(0)=0\right] d w \\
&= 1 / \lambda^{\prime}-D_{-\lambda^{\prime}}(0)\left\{\exp \left(-b^{2} / 4\right)\left[D_{-\lambda^{\prime}}(a)-D_{-\lambda^{\prime}}(-a)\right]\right. \\
&\left.+\exp \left(-a^{2} / 4\right)\left[D_{-\lambda^{\prime}}(b)-D_{-\lambda^{\prime}}(-b)\right]\right\} / \\
&\left\{\lambda^{\prime}\left[D_{-\lambda^{\prime}}(a) D_{-\lambda^{\prime}}(b)-D_{-\lambda^{\prime}}(-a) D_{-\lambda^{\prime}}(-b)\right]\right\} .
\end{aligned}
$$

Using (5.6), and letting $\beta=1$, this expression can be shown to include as subcases $(-b \rightarrow-\infty, b=a)$,

$$
\begin{aligned}
& \begin{aligned}
\int_{0}^{\infty} e^{-\lambda t} P\left[\sup _{0 \leqq s \leqq t} x(s)<a \mid x(0)=0\right] d t \\
=1 / \lambda\left\{1-\exp \left(-a^{2} / 4\right) D_{-\lambda}(0) / D_{-\lambda}(-a)\right\}, \\
\int_{0}^{\infty} e^{-\lambda t} P\left[\max _{0 \leqq s \leqq t}|x(s)|<a \mid x(0)=0\right] d t \\
=1 / \lambda\left\{1-\exp \left(-a^{2} / 4\right) 2 D_{-\lambda}(0) /\left[D_{-\lambda}(a)+D_{-\lambda}(-a)\right]\right\} .
\end{aligned}
\end{aligned}
$$

Expressions (5.8) and (5.9) are contained in [22], and [7], respectively. Another reference for (5.8) and (5.9) is [4] by Bellman and Harris. Equation (5.7) can also be derived from [7].

It appears very difficult to invert (5.7), (5.8), and (5.9), but formulas similar to (5.6), and tables of inverse Laplace transforms involving Weber functions, such as [1, pp. 289-91], may make future inversion possible.

VI. Proofs of theorems. Theorem 1 is similar to Theorem 3 of [3], but because it uses Gaussian-Markov functions $E\{F[x] \mid x(s)=x, x(t)=y\} p(x, s ; y, t)$ rather than $E\{F[x] \mid x(s)=x\}$ the present analysis is more delicate and not capable of convenient shortening by references to the earlier proof.

LEMMA 6.1. Assume $u$ and $v$ satisfy (2.3)-(2.5), and $A(s, t)$ is as in (2.2). Let $w(t)=u(t) / v(t), 0 \leqq t \leqq T$. Assume that $0<g \leqq v(t) \leqq G, 0 \leqq t \leqq T$, and

$$
M \geqq w^{\prime}(t) \geqq L>0, \quad 0 \leqq t \leqq T .
$$

Then the following results hold:

$$
\begin{gathered}
A(s, t) \in \downarrow \text { as a function of } s, 0 \leqq s \leqq t, \\
A(\tau, t)>g^{2}\left[w(t)-w\left(t_{0}\right)\right]>0, \quad s \leqq \tau \leqq t_{0}<t, \\
\int_{s}^{t}[A(s, w)]^{-1 / 2} d w<2[w(t)-w(s)]^{1 / 2} / g L \leqq 2[w(T)]^{1 / 2} / g L, \\
\int_{s}^{t}[A(w, t)]^{-1 / 2} d w<2[w(t)-w(s)]^{1 / 2} / g L \leqq 2[w(T)]^{1 / 2} / g L, \\
\int_{s}^{t}[A(s, w) A(w, t)]^{-1 / 2} d w<[w(t)-w(s)]^{2} \Gamma(3 / 2) \Gamma(3 / 2) / g^{2} L \Gamma(3) .
\end{gathered}
$$


Proof. $A(s, t)=v^{2}(t)[w(t)-w(s)], 0 \leqq s \leqq t \leqq T$. Conclusions (1) and (2) now follow by the hypotheses on $u$ and $v$. Results (3) and (4) are similar to a calculation in $[3$, p. 795]. Finally

$$
\begin{aligned}
\int_{s}^{t}[A(s, w) A(w, t)]^{-1 / 2} d w & \leqq \int_{w(s)}^{w(t)}[(y-w(s))(w(t)-y)]^{-1 / 2} / g^{2} L d y \\
& <[w(t)-w(s)]^{2} \Gamma(3 / 2) \Gamma(3 / 2) / g^{2} L \Gamma(3) .
\end{aligned}
$$

LEMMA 6.2. Under the hypotheses of Theorem $1, \partial r / \partial y$ and $\partial^{2} r / \partial y^{2}$ are continuous for $0 \leqq s<t \leqq T, y \in\{(-\infty, \infty)-E\}$. $\partial r / \partial y$ is also continuous if $y \in E$.

Proof. The conditions imposed on $\theta$ insure that $r$ exists for $R: 0 \leqq s<t \leqq T$, $-\infty<x, y<\infty$. From [8], the function $r$ satisfies the integral equation

$$
r(x, s \mid y, t)=p(x, s ; y, t)+\int_{s}^{t} \int_{-\infty}^{\infty} r(x, s \mid \alpha, \tau) \theta(\alpha, \tau) p(\alpha, \tau ; y, t) d \alpha d \tau
$$

Assume $|\theta(y, t)| \leqq M,(t, y) \in R, y \notin E$. Then (1.5) yields

$$
|r(x, s \mid y, t)| \leqq e^{t M} p(x, s ; y, t) .
$$

Let $G(y, t)$ equal the iterated integral in (6.1) and $f_{n}(y, t)$ equal the same integral with the outer limits of integration replaced by $s+1 / n$ and $t-1 / n$ for

$$
n>\max (1,1 / t-s) \text {. }
$$

For each $n,(\partial / \partial y) f_{n}(y, t)$ may be obtained by differentiation under the integral sign since the integral converges uniformly in any interval about $y$. Also $(\partial / \partial y) f_{n}(y, t)$ is continuous in $(y, t)$ for each $n$. Let

$$
g(y, t)=\int_{s}^{t} \int_{-\infty}^{\infty} \frac{\partial}{\partial y} p(\alpha, \tau ; y, t) \theta(\alpha, \tau) r(x, s \mid \alpha, \tau) d \alpha d \tau
$$

Observe that

$$
\left|g(y, t)-\frac{\partial}{\partial y} f_{n}(y, t)\right| \leqq\left|\int_{s}^{s+1 / n} \int_{-\infty}^{\infty} \frac{\partial}{\partial y} p \theta r d \alpha d \tau\right|+\left|\int_{t-1 / n}^{t} \int_{-\infty}^{\infty} \frac{\partial}{\partial y} p \theta r d \alpha d \tau\right|
$$

Lemma 6.1 shows that the second quantity, say $\left|I_{2}\right|$, is bounded by a constant independent of $y$.

$$
\left|I_{2}\right| \leqq \frac{M e^{t M} G / g}{\sqrt{(2 \pi g)^{2}(w(t-1 / n)-w(s))}} \int_{t-1 / n}^{t} \int_{-\infty}^{\infty} \frac{|z| e^{-z^{2} / 2}}{\sqrt{A(\tau, t)}} d z d \tau<\infty
$$

A similar argument applies to $\left|I_{1}\right|$.

Hence the sequence $\left\{(\partial / \partial y) f_{n}(y, t)\right\}$ converges uniformly to $g(y, t)$ in any interval about $y$. Hence $(\partial / \partial y) G(y, t)$ exists, is continuous, and equals $g(y, t)$ in $R$. 
Thus $(\partial / \partial y) r(x, s \mid y, t)$ exists, is continuous in $R$ and

$$
\frac{\partial r}{\partial y}(x, s \mid y, t)=-\frac{[y-x C(t, s)]}{A(s, t)} p(x, s ; y, t)+g(y, t),
$$

where we define $C(a, b)=v(a) / v(b), 0 \leqq a, b \leqq T$.

With a change of variables, and Lemma 6.1, $|g(y, t)|$ may be proved bounded, say by $K$, independent of $y$ and $t$.

If we assume that $\left|\theta_{y}(y, t)\right| \leqq B,(y, t) \in R, y \notin E$, then it follows that

$$
\begin{gathered}
\left|\frac{\partial}{\partial y}\{\theta[(y-z \sqrt{A(\tau, t)}) C(\tau, t), \tau] r[x, s \mid(y-\sqrt{A(\tau, t)} z) C(\tau, t), \tau]\}\right| \\
\leqq\left\{M|(y-z \sqrt{A(\tau, t)}) C(\tau, t)-x C(\tau, s)| / A(s, \tau)+B e^{t M}\right\} \\
\cdot p(x, s ;(y-z \sqrt{A(\tau, t)}), \tau)+M K .
\end{gathered}
$$

Using (6.4) and a lengthy argument similar to proving $(\partial / \partial y) G(y, t)=g(y, t)$, we obtain

$$
\frac{\partial^{2} G}{\partial y^{2}}(y, t)=-\int_{s}^{t} \int_{-\infty}^{\infty} \frac{z C(\tau, t)}{\sqrt{A(\tau, t)}} \frac{\exp \left(-z^{2} / 2\right)}{\sqrt{2 \pi}} \frac{\partial}{\partial y}(\theta r) d z d \tau
$$

where $\theta$ and $r$ are evaluated as in (6.4). This expression for $\partial^{2} G / \partial y^{2}$ is continuous for $(y, t) \in R, y \notin E$.

Since $\left(\partial^{2} / \partial y^{2}\right) p(x, s ; y, t)$ exists and is continuous in the strip $R, \partial^{2} r / \partial y^{2}$ exists and is continuous there, $y \notin E$, and $\partial^{2} r / \partial y^{2}=\partial^{2} p / \partial y^{2}+\partial^{2} G / \partial y^{2}$.

Proof of Theorem 1. As a first step in obtaining $\partial G / \partial t$ we shall rewrite $\partial G / \partial y$ to involve $(\partial / \partial y)[\theta r]$ rather than $\partial p / \partial y$. Since

$$
\frac{\partial}{\partial y} p(z, \tau ; y, t)=-C(\tau, t) \frac{\partial p}{\partial z}(z, \tau ; y, t)
$$

using integration by parts, the vanishing of $p$ for large $|z|$, the bound on $r$, and a change of variables we obtain

$$
\frac{\partial G}{\partial y}=\int_{s}^{t} \int_{-\infty}^{\infty} C(\tau, t) \frac{\exp \left(-z^{2} / 2\right)}{\sqrt{2 \pi}} \frac{\partial}{\partial y}[\theta r] d z d \tau
$$

where $\theta$ and $r$ are evaluated as in (6.4).

From (6.3), (6.5), and (6.6), an obvious integration yields

$$
\begin{aligned}
& \int_{s}^{t}[A(w)\left.\frac{\partial^{2} G}{\partial y^{2}}-B(w) \frac{\partial}{\partial y}[y G]\right] d w \\
&=-\int_{s}^{t} \int_{s}^{w} \int_{-\infty}^{\infty} \frac{\exp \left(-z^{2} / 2\right)}{\sqrt{2 \pi}} \frac{\partial}{\partial y}[\theta r] \\
& \quad \times\left\{\frac{A(w) z C(\tau, w)}{\sqrt{A(\tau, w)}}+y B(w) C(\tau, w)\right\} d z d \tau d w \\
&-\int_{s}^{t} B(w) G(y, w) d w .
\end{aligned}
$$


We now wish to apply Fubini's Theorem so that the iterated integration can be performed in the order $w, z, \tau$.

With $\theta$ and $r$ written in full in (6.4), a bound on $|(\partial / \partial y)[\theta r]|$ given by (6.4), and I denoting the triply iterated integral with the integrand replaced by its absolute value, we have $I \leqq J_{1}+J_{2}+J_{3}$ where the bound on $|(\partial / \partial y)[\theta r]|$ has been split into three parts. By Lemma 6.1 $J_{2}$ and $J_{3}$ are finite. To handle $J_{1}$, we will use a technique of E. Nelson [17].

There exists a constant $N$ such that

$$
N \geqq \frac{|(y-z \sqrt{A(\tau, w)}) C(\tau ; w)-x C(\tau, s)|^{3 / 2}}{A(s, \tau)^{1 / 4}} p(x, s ;(y-z \sqrt{A(\tau, w)}) C(\tau, w), \tau)
$$

for all values of $z, y, x$, and $\tau$ such that $s<\tau$. Using (6.8) and an extension of (5), Lemma 6.1 to an exponent of $-3 / 4$, one can show that $J_{1}$ is finite.

Next we have

$$
\frac{\partial}{\partial w}[\theta r]=-\left[y B(w)+z \frac{A(w)}{\sqrt{A(\tau, w)}}\right] \frac{\partial}{\partial y}[\theta r]
$$

where $\theta$ and $r$ are given in full in (6.4) with $t$ replaced by $w$.

Hence, (6.7) becomes

$$
\begin{aligned}
& \int_{s}^{t} {\left[A(w) \frac{\partial^{2} G}{\partial y^{2}}-B(w) \frac{\partial}{\partial y}[y G]\right] d w } \\
& \quad=\int_{s}^{t} \int_{-\infty}^{\infty} \int_{\tau}^{t} \frac{\exp \left(-z^{2} / 2\right)}{\sqrt{2 \pi}} C(\tau, w) \frac{\partial}{\partial w}[\theta r] d w d z d \tau-\int_{s}^{t} B(w) G(y, w) d w .
\end{aligned}
$$

If I represents the iterated integral on the right side of (6.9), by performing an integration by parts on the inner integral, then changing variables, using the definition of $G(y, t)$, changing variables again, and using Fubini's Theorem,

$$
\mathrm{I}=G(y, t)-\int_{s}^{t} \theta[y, \tau] r(x, s \mid y, \tau) d \tau+\int_{s}^{t} B(w) G(y, w) d w
$$

Thus it is clear that $\partial G(y, t) / \partial t$ exists and is continuous on $(0, T) \times\{(-\infty, \infty)-E\}$ and we have

$$
A(t) \frac{\partial^{2} G}{\partial y^{2}}-B(t) \frac{\partial}{\partial y}[y G]=\frac{\partial G(y, t)}{\partial t}-\theta[y, t] r(x, s \mid y, t)
$$

Furthermore

$$
A(t) \frac{\partial^{2} p}{\partial y^{2}}-B(t) \frac{\partial}{\partial y}[y p]=\frac{\partial p}{\partial t}
$$

in the strip, so that using (6.1), equation (1.1) is satisfied.

For the boundary condition (1.2) let $g(x)$ be a continuous bounded function, 
$-\infty<x<\infty$. By (6.1), we have

$$
\begin{aligned}
\int_{-\infty}^{\infty} g(x) r(x, s \mid y, t) d x & =\int_{-\infty}^{\infty} g(x) p(x, s ; y, t) d x \\
& +\int_{-\infty}^{\infty} \int_{s}^{t} \int_{-\infty}^{\infty} g(x) r(x, s \mid \alpha, \tau) \theta(\alpha, \tau) p(\alpha, \tau ; y, t) d \alpha d \tau d x .
\end{aligned}
$$

If I denotes the last integral, by (6.2) and the Chapman-Kolmogorov equation, [10],

$$
|\mathrm{I}| \leqq \int_{-\infty}^{\infty} \int_{s}^{t}|g(x)| e^{\tau M} M p(x, s ; y, t) d \tau d x .
$$

Since $p$ becomes singular at $s=t, x=y$,

$$
\lim _{t \rightarrow s+} \int_{-\infty}^{\infty} g(x) p(x, s ; y, t) d x=g(y)
$$

and a similar result holds for $|g(x)|$. Furthermore, from (6.12) and the boundedness of $g$,

$$
\lim _{t \rightarrow s^{+}} \sup |\mathrm{I}| \leqq M|g(y)| \lim _{t \rightarrow s^{+}} \sup \int_{s}^{t} e^{\tau M} d \tau=0 .
$$

Using (6.11), (6.13), and the zero limit for I, the boundary condition (1.2) follows.

Boundary condition (1.3) follows from (6.2) and the fact that

$$
\lim _{|y| \rightarrow \infty} p(x, s ; y, t)=0 .
$$

Conditions (1.4) were established in Lemma 6.2.

The uniqueness follows from [8], as stated in the summary.

The backwards system (3.3)-(3.6) is proved along similar lines starting with the integral equation

$$
r(x, s \mid y, t)=p(x, s ; y, t)+\int_{s}^{t} \int_{-\infty}^{\infty} p(x, s ; \alpha, \tau) \theta(\alpha, \tau) r(\alpha, \tau \mid y, t) d \alpha d \tau .
$$

The term $x(\partial r / \partial x)$ in the backwards equation rather than the term $(\partial / \partial y)[y r]$ in the forward equation eliminates the need for an argument similar to that following (6.9).

Proof of Theorem 2. By Theorem 1, and the stationarity of $p(x, s ; y, t), Q(y, t)$ satisfies (1.1) with coefficients $A, B,-u V(y)$, for $0<t<T, y \in\{(-\infty, \infty)-E\}$. Multiplying by $e^{-\lambda t}$ and integrating on $t$ between 0 and $T$, we have the existence of a function $Q(y, t)$ which satisfies the equation

$$
\begin{aligned}
A \int_{0}^{T} e^{-\lambda t} \frac{\partial^{2} Q(y, t)}{\partial y^{2}} d t-B \int_{0}^{T} e^{-\lambda t} \frac{\partial}{\partial y}[y Q(y, t)] d t & \\
& -u V(y) \int_{0}^{T} e^{-\lambda t} Q(y, t) d t=\int_{0}^{T} e^{-\lambda t} \frac{\partial Q(y, t)}{\partial t} d t .
\end{aligned}
$$


We will now show that it is possible to let $T \rightarrow \infty$ and interchange integration and differentiation.

Let $\hat{p}(w)=\int_{0}^{\infty} e^{-\lambda t} p(0,0 ; w, t) d t$. The existence of $\hat{p}$ is subsumed in the proof below of (3.11). This fact plus the conditions on $V$ ensure that the Laplace transform of $Q$ exists. To show that $d \hat{Q} / d y$ exists and equals $\int_{0}^{\infty} e^{-\lambda t} d Q(y, t) / \partial y$ we essentially need to show (3.11).

From the bound on $\partial r / \partial y$ established in Lemma 6.2, (6.8), and Lemma 6.1

$$
\begin{aligned}
& \left|\int_{0}^{\infty} e^{-\lambda t} \frac{\partial}{\partial y} Q(y, t) d t\right| \\
& \leqq \int_{0}^{\infty} e^{-\lambda t} \frac{|y|}{A(0, t)} p(0,0 ; y, t) d t+\frac{K}{\lambda} \\
& \leqq \int_{0}^{\infty} e^{-\lambda t} \frac{\max \left(1,|y|^{3 / 2}\right)}{A(0, t)} p(0,0 ; y, t) d t+\frac{K}{\lambda} \\
& <N\left\{\int_{0}^{1}[A(0, t)]^{-3 / 4} d t+\int_{1}^{\infty} e^{-\lambda t}[A(0, t)]^{-3 / 4} d t\right\}+\frac{K}{\lambda} \\
& \leqq N\left\{4[u(1) / v(1)]^{1 / 4} / L g^{3 / 2}+e^{-\lambda}[u(1) / v(1)-u(0) / v(0)]^{-3 / 4} / \lambda g^{3 / 2}\right\}+\frac{K}{\lambda} .
\end{aligned}
$$

Thus

$$
\frac{d \hat{Q}(y)}{d y}=\int_{0}^{\infty} e^{-\lambda t} \frac{\partial}{\partial y} Q(y, t) d t \text { and }\left|\frac{d \hat{Q}(y)}{d y}\right|<\text { bound of (6.15) }
$$

which establishes (3.11).

Since $Q(y, t)$ is continuous for $0<t \leqq 1,-\infty<y<\infty$ and bounded for $1<t<\infty$, $-\infty<y<\infty$,

$$
\int_{0}^{\infty} e^{-\lambda t} \frac{\partial Q(y, t)}{\partial t} d t=\lambda \hat{Q}(y)-Q(y, 0+) .
$$

By using (6.2), (2.1), and (2.2), $\lim _{t \rightarrow 0+} Q(y, t)=0$ for $y \neq 0$.

Using equation (6.14), for any point $y_{0} \neq 0$,

$$
A \int_{0}^{\infty} e^{-\lambda t} \frac{\partial^{2} Q(y, t)}{\partial y^{2}} d t=\int_{0}^{\infty} e^{-\lambda t}\left\{B y \frac{\partial Q}{\partial y}+Q+\frac{\partial Q}{\partial t}+u V(y)\right\} d t
$$

converges uniformly for $\left|y-y_{0}\right| \leqq \delta$, some $\delta>0$. Hence

$$
\frac{d^{2} \hat{Q}(y)}{d y^{2}}=\int_{0}^{\infty} e^{-\lambda t} \frac{\partial^{2} Q(y, t)}{\partial y^{2}} d t .
$$

Thus equation (6.14) transforms to equation (3.7) and the solution $Q$ of (6.14) transforms to the solution $\hat{Q}$ of (3.7).

Using dominated convergence and (1.4) gives (3.10).

To establish (3.8), we first bound $|\hat{Q}(y)|$ as in (6.15). Next we use the identity $(\varepsilon>0, \delta>0)$

$$
\int_{-\varepsilon}^{\varepsilon} \int_{\delta}^{\infty}\left\{\frac{\partial Q}{\partial t}-A \frac{\partial^{2} Q}{\partial y^{2}}+B \frac{\partial}{\partial y}[y Q]+u V(y) Q\right\} e^{-\lambda t} d t d y=0
$$


an integration by parts, and previous formulas to obtain

$$
\begin{array}{r}
-\lim _{\delta \rightarrow 0+} \int_{-\varepsilon}^{\varepsilon} e^{-\lambda \delta} Q(y, \delta) d y+\int_{-\varepsilon}^{\varepsilon}[\lambda+u V(y)] \hat{Q}(y) d y \\
-\left.A \hat{Q}^{\prime}(y)\right|_{-\varepsilon} ^{\varepsilon}+\left.B[y \hat{Q}]\right|_{-\varepsilon} ^{\varepsilon}=0 .
\end{array}
$$

We now use (6.1) to obtain

$$
\lim _{t \rightarrow 0+} \int_{-\varepsilon}^{\varepsilon} Q(y, t) d y=1 \quad \text { for all } \varepsilon>0 .
$$

Clearly $\lim _{t \rightarrow 0+} \int_{-\varepsilon}^{\varepsilon} p(0,0 ; y, t) d y=1$. Using the bound on $\theta,(6.2)$, and the Chapman-Kolmogorov equation,

$\left|\int_{|y| \leqq \varepsilon} \int_{0}^{t} \int_{-\infty}^{\infty} r(0,0 \mid \alpha, \tau) \theta(\alpha, \tau) p(\alpha, \tau ; y, t) d \alpha d \tau d y\right| \leqq \int_{|y| \leqq \varepsilon} p(0,0 ; y, t) d y M e^{t M} t$, which approaches 0 as $t \rightarrow 0+$. Therefore, condition (6.17) is satisfied. We then let $\varepsilon \rightarrow 0$ in (6.16) and use the boundedness of $V$ and $\hat{Q}$ to obtain (3.8).

We obtain (3.9) from an extension of (6.2) due to the form of $\theta$, and dominated convergence.

Uniqueness will be proved by an argument analogous to one used by $\mathrm{M}$. Kac in [15]. Assume there are two solutions $\hat{Q}_{1}$ and $\hat{Q}_{2}$ to (3.7)-(3.11). If $\phi=\hat{Q}_{1}-\hat{Q}_{2}$, then $\phi$ satisfies (3.7), (3.9), (3.10), $\phi^{\prime}(0+)-\phi^{\prime}(0-)=0,\left|\phi^{\prime}(y)\right|<2 C+2 D / \lambda, y \neq 0$. Multiplying (3.7) by $\phi$ and integrating between $-E$ and $F(E>0, F>0)$, we obtain

$$
A \int_{-E}^{F} \phi^{\prime \prime} \phi d y-B \int_{-E}^{F} y \phi^{\prime} \phi d y=\int_{-E}^{F}[B+u V(y)+\lambda] \phi^{2} d y .
$$

After integrating each of the first two integrals by parts, we let $E \rightarrow \infty, F \rightarrow \infty$ and use the hypotheses on $\phi$ to obtain

$$
-A \int_{-\infty}^{\infty}\left[\phi^{\prime}\right]^{2} d y=\int_{-\infty}^{\infty}[\lambda+u V(y)] \phi^{2} d y
$$

Since $A>0, \lambda+u V(y)>0$, we must have $\phi(y) \equiv 0,-\infty<y<\infty$.

Acknowledgements. This paper was started at the University of Minnesota. The author would like to thank Professor Glen Baxter for helpful suggestions in writing this paper, and the referee for several improvements.

\section{REFERENCES}

1. Bateman Manuscript Project, Tables of integral transforms, Vol. 1, McGraw-Hill, New York, 1953.

2. - Higher transcendental functions, Vol. 2, McGraw-Hill, New York, 1953.

3. John A. Beekman, Gaussian processes and generalized Schroedinger equations, J. Math. Mech. 14 (1965), 789-806.

4. R. Bellman and T. Harris, Recurrence times for the Ehrenfest model, Pacific J. Math. 1 (1951), 179-193. 
5. R. H. Cameron, The generalized heat flow equation and a corresponding Poisson formula, Ann. of Math. 59 (1954), 434-462.

6. T. M. Cherry, Expansions in terms of parabolic cylinder functions, Proc. Edinburgh Math. Soc. (2) 8 (1949), 50-65.

7. D. A. Darling and A. J. F. Siegert, The first passage problem for a continuous Markov process, Ann. Math. Statist. 24 (1953), 624-639.

8. - Integral equations for the characteristic functions of certain functionals of multidimensional Markoff processes, Rand Report, P-429, Rand Corporation, Santa Monica, California, 1955.

9. J. L. Doob, Heuristic approach to the Kolmogorov Smirnov theorems, Ann. Math. Statist. 20 (1949), 393-403.

10. - Stochastic processes, Wiley, New York, 1953.

11. R. K. Getoor, Additive functionals of a Markov process, Pacific J. Math. 7 (1957), 1577-1591.

12. - Markov operators and their associated semi-groups, Pacific J. Math. 9 (1959), 449-472.

13. M. Kac, Random walk and the theory of Brownian motion, Amer. Math. Monthly 54 (1947), 369-391.

14. - On deviations between theoretical and empirical distributions, Proc. Nat. Acad. Sci. U.S.A. 35 (1949), 252-257.

15. — On distributions of certain Wiener functionals, Trans. Amer. Math. Soc. 65 (1949), 1-13.

16. - On sume connections between probability theory and differential and integral equations, Proc. Second Berkeley Symposium, pp. 189-215, Univ. of Calif. Press, Berkeley, Calif., 1951.

17. Edward $O$. Nelson, $A$ solution of the generalized heat flow equation in a bounded region as a Wiener integral, Ph.D. Thesis, Univ. of Minnesota, Minneapolis, 1959.

18. J. M. H. Olmsted, Advanced calculus, Appleton Century Crofts, New York, 1961.

19. L. S. Ornstein and G. E. Uhlenbeck, On the theory of the Brownian motion, Phys. Rev. 36 (1930), 823-841.

20. D. Ray, On the spectra of second order differential operators, Trans. Amer. Math. Soc. 77 (1954), 299-321.

21. M. Rosenblatt, On a class of Markov processes, Trans. Amer. Math. Soc. 71 (1951), $120-135$.

22. A. J. F. Siegert, On the first passage time probability problem, Phys. Rev. 81 (1951), 617-623.

23. I. Vincze, On two-sample tests based on order statistics, Proc. Fourth Berkeley Symposium, Vol. 1, pp. 695-705, Univ. of Calif. Press, Berkeley, Calif., 1961.

24. E. T. Whittaker and G. N. Watson, A course of modern analysis, Cambridge Univ. Press, Cambridge, 1935.

25. Eugene Wong, The construction of a class of stationary Markov processes, Proc. Sympos. Appl. Math., Vol. 16, pp. 264-276, Amer. Math. Soc., Providence, R. I., 1964.

Ball State University, MUNCIE, INDIANA 